Statistica Neerlandica (2010) Vol. 64, nr. 1, pp. 1-18

doi:10.1111/j.1467-9574.2009.00440.x

\title{
Modeling and prediction of surgical procedure times
}

\author{
Pieter S. Stepaniak* \\ Institute of Health Policy and Management, Erasmus University \\ Rotterdam, P.O. Box 1738, 3000 DR Rotterdam, The Netherlands \\ Christiaan Heij \\ Econometric Institute, Erasmus School of Economics, Erasmus \\ University Rotterdam, The Netherlands \\ and Guus de Vries \\ Institute of Health Policy and Management, Erasmus University \\ Rotterdam, P.O. Box 1738, 3000 DR Rotterdam, The Netherlands
}

\begin{abstract}
Accurate prediction of medical operation times is of crucial importance for cost-efficient operation room planning in hospitals. This paper investigates the possible dependence of procedure times on surgeon factors like age, experience, gender and team composition. The effect of these factors is estimated for over 30 different types of medical operations in two hospitals, by means of ANOVA models for logarithmic case durations. The estimation data set contains about 30,000 observations from 2005 to 2008 . The relevance of surgeon factors depends on the type of operation. The factors found most often to be significant are team composition, experience and time of the day. Contrary to widespread opinions among surgeons, gender has nearly never a significant effect. By incorporating surgeon factors, the accuracy of out-of-sample prediction of case durations of about 1250 surgical operations in 2009 is improved by up to more than $15 \%$ compared with current planning procedures.
\end{abstract}

Keywords and Phrases: operation room, surgeon factors, lognormal distribution, ANOVA model, planning, European hospital, healthcare management, current procedure terminology.

\section{Introduction}

Operating rooms (ORs) are among the most expensive surgical resources in hospitals (Vissers and Beech, 2005). In an era of cost-constrained health care, efficiency increases if a larger number of surgical operations can be performed within the available OR time (StePaniak et al., 2009b). The OR management of medical

\footnotetext{
*stepaniak@bmg.eur.nl 
institutions needs to balance the costs of reserving too much time, with resulting idle time of the OR, against the costs of reserving too little time. In the last case, the OR schedule must be modified, resulting in an increased demand for anesthesiologists, nurses and support staff. Therefore, accurate prediction of case durations helps in effective OR scheduling, it reduces waiting times for patients and idle times of medical and other staff, and thereby it improves the quality of health care delivered in other services throughout the hospital.

Surgical procedure times are inherently unpredictable, and the amount of uncertainty varies greatly among different types of operations. Hospitals employ standard classifications of operations, in terms of the so-called current procedure terminologies (CPTs). Apart from the CPT, surgeon factors are the primary source of variation in case durations, as shown in Strum, MaY and VARges (2000a) and STRUM et al. (2000b).

The purpose of this paper is to quantify the effect of surgeon factors on case durations and to exploit these factors to improve case duration predictions. The empirical analysis is based on extensive databases of surgical operations in two teaching hospitals in The Netherlands. The OR management in these two hospitals often receives arguments brought forward by surgeons, anesthetists and OR staff, as to why surgical cases should be planned shorter or longer than usual due to a range of factors. The factors mentioned most frequently to slow down procedure times are the following: composition of the surgical team (presence of residents, that is, physicians receiving specialized clinical training), lack of experience (low recent work rate for this CPT), gender (female surgeons would be more precise and more careful and hence slower), age (younger surgeons are less experienced) and time of the day (fatigue in the afternoon). Some of these factors have been analyzed before for hospitals in the USA, for instance, in STRUm et al. (2000b). As labor regulations and working habits are quite different in Europe, it is of interest to study the effect of these factors within a European setting.

The main results are the following. For several CPTs, some of the factors contribute significantly (at the $1 \%$ significance level) to operation times. This holds true most notably for relatively complex surgical operations, for instance, those involving endoscopic and laparoscopic procedures. Team composition, work rate and time of the day are the most commonly relevant factors. Age matters only for two CPTs, and gender for none of the CPTs (and at the 5\% significance level only for a single $\mathrm{CPT}$, cataract in hospital A, where female surgeons work faster than their male colleagues). The practical relevance of these factors is demonstrated by improved out-of-sample prediction of case durations for 2009. Compared with current OR planning procedures, which are based on the last ten cases of each CPT, the accuracy is improved by $10-15 \%$. Even if the more advanced three-parameter lognormal model for case durations is taken as a benchmark, incorporation of significant surgeon factors leads to improvements of the same order of magnitude.

The paper has the following structure. Section 2 presents the data, and section 3 discusses the statistical model for case durations. The results in terms of relevant (c) 2009 The Authors. Journal compilation (c) 2010 VVS. 
factors and the gains in predictive accuracy are described in section 4, and section 5 concludes.

\section{Data}

\subsection{Surgical procedure times}

The data are obtained from surgical databases of two large teaching hospitals in The Netherlands, covering about 100,000 operations in the period from January 2005 to August 2009. The data from 2005 to 2008 are used in estimation, leaving out the data of 2009 for predictive evaluation purposes. The two hospitals, that will be labeled as A and B, differ in several aspects, such as covered specializations, organizational structure, OR protocols, OR logistics and intensity of teaching. Therefore, the two hospitals will be analyzed separately but with similar methods.

For each operation, the database contains information on the type of operation (the CPT-anesthesia combination), on the procedure and surgical times, and on several surgeon factors (as will be discussed in section 2.2). The procedure time is defined as the time passing from entry into the operating suite to leaving the OR. This includes the surgical time, that is, the time passing from incision to closure of the wound. The attention will be focused on procedure times, as these are the relevant durations for OR planning. These times will also be denoted as surgical procedure times, indicating that these times include the surgical operation itself as well as the required OR procedures preceding and following the operation.

For the period 2005 to 2008, the database of hospital A contains over 44,000 cases for nearly 1200 CPT-anesthesia combinations, with total OR time of about 50,000 hours. For various reasons, the actually employed data set is much smaller and contains 17,516 cases for 29 CPT-anesthesia combinations and a total OR time of about 20,000 hours. The main reason for this data reduction is that CPTs are excluded if they occur relatively infrequently or if they are always performed under similar circumstances. More precisely, in order to be included in the analysis, a CPT-anesthesia combination should exhibit sufficient variation in surgeon factors to allow for an analysis of the effect of these factors. Therefore, for every CPTanesthesia combination, the imposed minimal requirements are at least 150 cases in total and at least 25 cases for every surgeon involved. Further, about $15 \%$ of the cases consist of composite operations involving multiple CPTs. These operations are excluded to avoid possible confounding factors, following STRUм et al. (2000a). Composite operations do not only occur rather infrequently in a fixed composition, but other factors such as the order of the operations may also affect the composite case durations. Minor other reasons for exclusion are operations with incomplete data (less than 1\%), and special operations like donor procedures and operations not started or not completed (less than $0.1 \%$ ).

A similar data selection strategy is followed for hospital B. This database contains about 42,000 cases for about 1000 CPT-anesthesia combinations, with a (C) 2009 The Authors. Journal compilation (c) 2010 VVS. 
total OR time of about 45,000 hours. The actually employed data set, after applying the selection strategy discussed before, contains 12,030 cases for 25 CPT-anesthesia combinations and a total OR time of about 16,000 hours.

The total number of included CPT-anesthesia combinations in hospitals A and B is 32, with 22 common ones for hospitals A and B, seven for hospital A alone and three for hospital B alone. Table 1 shows the included CPTs and contains information on the procedure times. The last four columns show the total number of surgeons and residents involved in each CPT, as well as the number of cases performed in the morning and in the afternoon.

\subsection{Surgical factors}

The literature review of DEXTER et al. (2008) identifies 48 papers reporting significant factors affecting the perioperative time, that is, the total time required for a patient's surgical procedure, including ward admission, anesthesia, surgery and recovery. There are multiple reports of the effects on OR times of operative procedures, perioperative team composition including primary surgeon, type of anesthetic and patient characteristics, in this sequence of importance. STRUM et al. (2000a, b) mention surgeon factors as the single most important source of variability in surgical procedure times. Other, secondary sources of variability mentioned in their study are the type of anesthesia, age and gender of the patient and American Society of Anesthesiologists risk class. The age of the surgeon is mentioned in VAN HoudenHOVEN (2007).

As described in section 1, several of these surgeon factors were also brought forward by surgeons, anesthetists and OR managers in hospitals A and B. In total, the following five factors will be taken into account.

\subsubsection{Gender}

A popular belief is that female surgeons are more precise and more careful in performing operations, resulting in longer case durations. The gender of the surgeon is indicated by the dummy variable 'Female' (with value 1 for females and 0 for males). For the CPTs of Table 1, the total numbers of female and male surgeons in hospital A are respectively, 7 and 23, and in hospital B these numbers are 7 and 18.

\subsubsection{Age}

In general, older surgeons are more experienced and they may therefore work more efficiently. This effect is mentioned, for instance, in VAN Houdenhoven (2007). It could also be that surgeons work the fastest in the middle period of their career, as older surgeons may become tired more quickly. However, because of the limited number of surgeons, a distinction in two age categories is preferred. The age of surgeons who are active in hospitals A and B ranges between 30 and 60 years. The (c) 2009 The Authors. Journal compilation (c) 2010 VVS. 


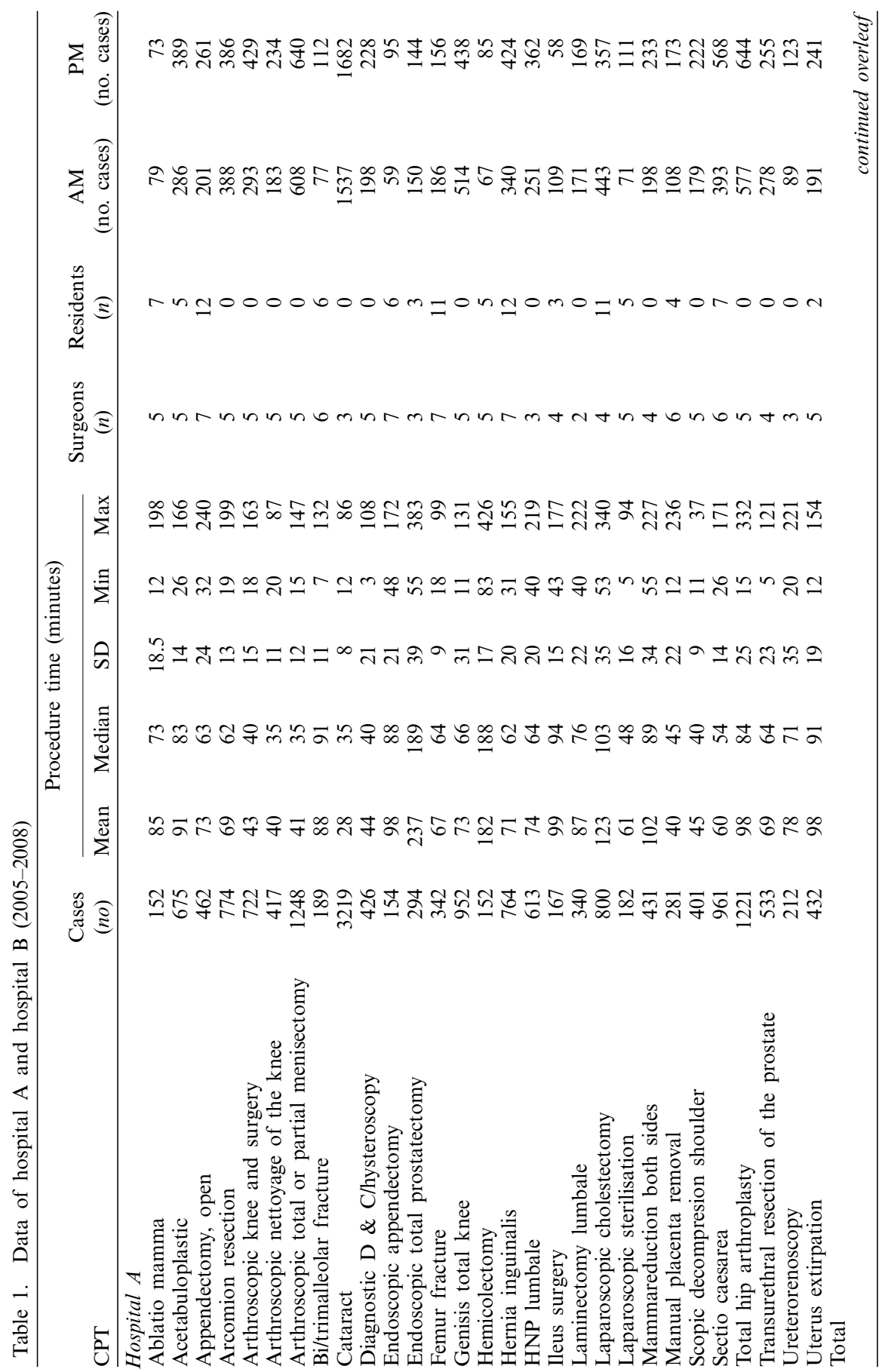




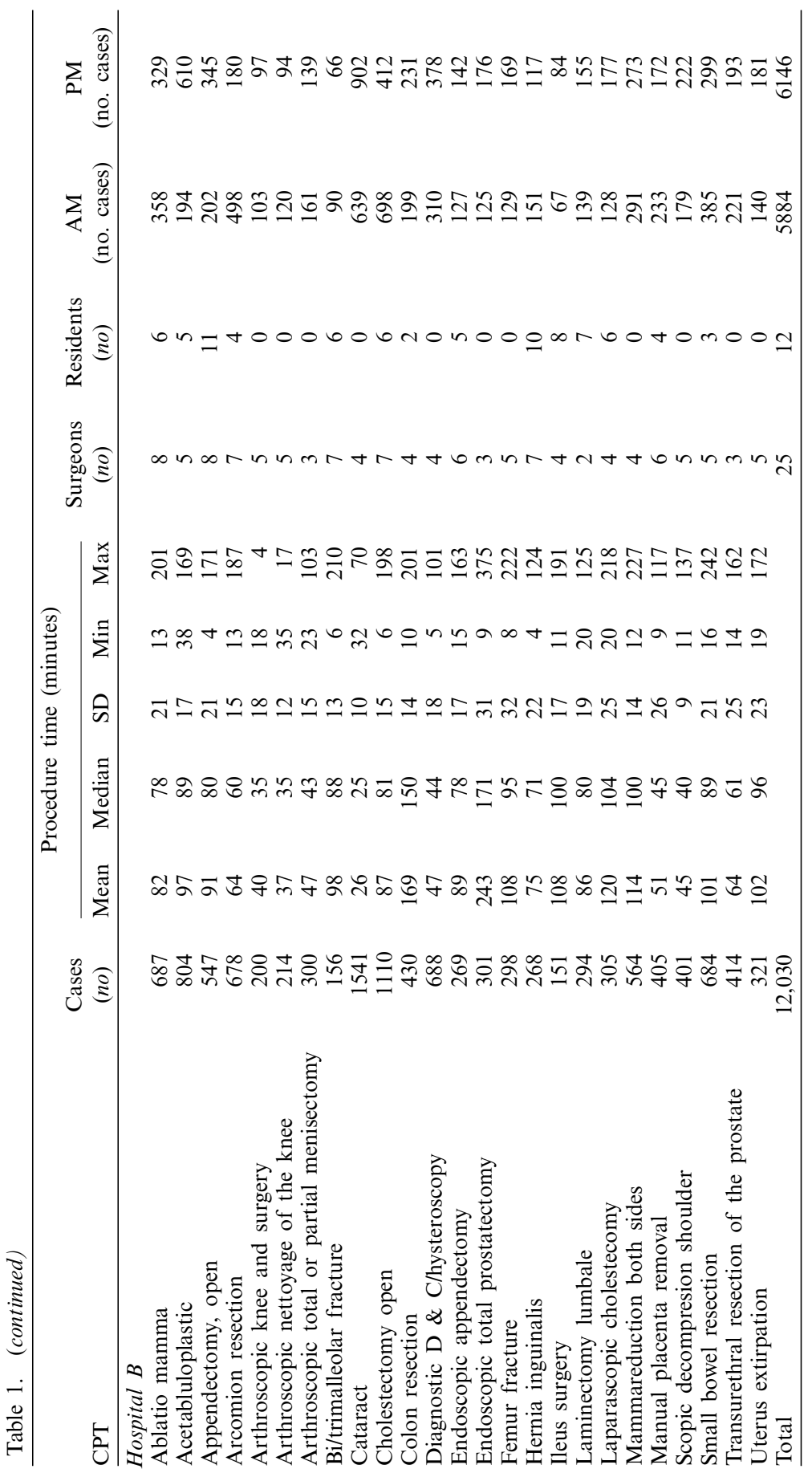


two age groups are indicated by the dummy variable 'Age', with value 1 if 45 or above and 0 if younger than 45 . For the CPTs of Table 1, the total numbers of surgeons above and below 45 years of age are respectively, 14 and 16 in hospital A, and in hospital B these numbers are, 13 and 12. For a team of surgeons performing an operation, the age is defined as the age of the oldest surgeon in the team.

\subsubsection{Work rate}

For a given CPT and surgeon, the work rate is related to the number of similar operations that this surgeon has performed in the recent past. A higher work rate means that the surgeon is more experienced in this kind of operation and that case durations may become shorter (Strum et al., 2000a). Again, because of the limited number of surgeons, a distinction in two classes of work rate is preferred. The work rate is defined to be high if the surgeon performed a similar CPT at most three weeks ago, and it is defined to be low if this was more than three weeks ago. This rate is indicated by the dummy variable 'Work rate', with value 1 for a high rate and 0 for a low rate. For the CPTs of Table 1, the percentage of operations with a high work rate is 81 for hospital $\mathrm{A}$ and 84 for hospital B. For a team of surgeons performing an operation, the work rate is defined as the work rate of the leading surgeon of the team.

\subsubsection{Team}

For all procedures of Table 1, the OR surgeon team always consists of a surgeon who is assisted by at least one other surgeon or a resident. Residents are surgeons who receive specialized clinical training in the hospital. It is common belief that the presence of a resident has an increasing effect on case durations because the resident receives on-the-job training during the operation. The team composition is indicated by the dummy variable 'Team', with value 1 if the team consists of surgeons only and 0 if a resident is part of the team.

\subsubsection{Time of the day}

Some people work better in the morning, others in the afternoon, in the evening or at night. A recent study (ТАмм et al., 2009) shows differences in brain excitability, that is, people who say that they feel best during a certain part of the day tend to have a brain that is most easily excitable during that part of the day. As an operation is a team effort of the involved surgeons and assisting staff, it is not easy to combine the time of the day effect for each individual in a joint team effect. Still, it is of interest to know whether the time of the day has an effect on case durations. The time of an operation is indicated by the dummy variable 'Daytime', with value 1 for the afternoon (operations starting at $12.00 \mathrm{PM}$ or later) and 0 for the morning (operations starting before $12.00 \mathrm{PM}$ ). It might be that case durations are 
longer in the evening and at night, due to less availability of surgeons and staff. However, such operations are very rare in the two hospitals under consideration, and there is insufficient information to test for separate evening and night effects. Therefore, operations taking place during the evening or at night are excluded due to insufficient data.

\section{Model for surgical procedure durations}

\subsection{Distribution of case durations}

The literature on surgical procedure times deals nearly exclusively with the situation in the USA. Early results report a lognormal distribution for OR waiting times (Rossiter and Reynolds, 1963) and a normal (BARNoon and Wolfe, 1968) or lognormal (HANCOCK et al., 1988) distribution for OR case durations. Insight into the distribution of case durations have advanced markedly in the past decade (STRUM et al., 2000a, b, 2003, May, Strum and Vargas, 2000, Spangler et al., 2004). The empirical study of STRUM et al. (2000a) indicates a lognormal distribution of surgical procedure times. Strum et al. (2003) consider composite operations consisting of two different surgical procedures and conclude that the lognormal distribution fits such case durations better than the normal distribution.

As surgical procedures require a positive start-up time, the shifted lognormal distribution (also called the three-parameter $\operatorname{lognormal}$, written as $3-\log \mathrm{N}$ ) is used in Strum et al. (2000a) and, within an European context, in STEPaniak et al. (2009a). For the far majority of CPTs, this distribution provides a better fit than the normal and lognormal distributions. Let the procedure time (in minutes) of a given CPT be denoted by $T$, then the $3-\log N$ distribution for can be written as

$$
\log (T-\alpha)=\beta+\varepsilon, \quad \varepsilon \sim N\left(0, \sigma^{2}\right) .
$$

Here, $\alpha>0$ is the shift parameter and $\varepsilon$ denotes an unobserved random error term causing unpredictable variation. Stated otherwise, after shifting by $\alpha$, the logarithmic procedure times are normally distributed with mean $\beta$ and standard deviation $\sigma$. The procedure time is always larger than $\alpha$, and the median is equal to $\alpha+\exp (\beta)$.

The effect of surgeon factors on case durations is modeled by replacing $\beta$ in the above model by parameters depending on the factors, similar to what is done in analysis of variance (ANOVA) models. If all five factors discussed in section 2.2 are included, the model becomes

$$
\begin{aligned}
\log (T-\alpha)= & \beta_{P T}+\varepsilon, \quad \varepsilon \sim N\left(0, \sigma^{2}\right), \\
\beta_{P T}= & \beta_{0}+\beta_{1} \times \text { Gender }+\beta_{2} \times \text { Age }+\beta_{3} \times \text { Work rate } \\
& +\beta_{4} \times \text { Team }+\beta_{5} \times \text { Daytime. }
\end{aligned}
$$

We call this the ANOVA model. This model is estimated for each CPT and each hospital separately, allowing for different surgeon factor effects according to the hospital 
and the type of surgical procedure. Although it may be possible to cluster some of the CPTs in Table 1 in groups with identical parameters, this will not be pursued here because the OR planning system is based on individual CPTs. For a given CPT and hospital, the error terms associated with all corresponding case durations in the database are assumed to be independent and identically distributed.

The various hypotheses on surgeon factors discussed in section 2.2 can be expressed in terms of the following hypotheses on the parameters of the above model:

$$
\beta_{1}>0, \quad \beta_{2}<0, \quad \beta_{3}<0, \quad \beta_{4}<0 .
$$

Further, it is expected that surgeon factors become more important as the complexity of surgical procedures increases. A procedure is complex if it requires highly trained OR staff performing very specific operational procedures and if the risk of perioperative complications is larger than what is usual for routine procedures.

\subsection{Estimation and prediction}

For each CPT of Table 1, the ANOVA model for procedure times is estimated for both hospitals separately, using data from the period 2005-2008. Factors that do not vary are removed from the model. For instance, if all surgeons for a CPT are male, then the effect of gender cannot be estimated for this CPT. To start, all factors that do vary for the CPT are included in the model. Next, backward elimination is used for stepwise removal of insignificant factors. In the end, if all remaining factors are significant, each of the other factors is tested once more for significance when added to the other factors. In addition, the significance of interaction effects between the factors is tested (as none of these interactions is significant, these results will not be reported). All tests employ the same significance level, which is $10 \%, 5 \%$ or $1 \%$.

To evaluate the practical relevance of the identified significant surgeon factors, the models that are estimated with data for 2005-2008 are used to predict the case durations in the period from January to August 2009. The prediction model is kept fixed, even though the parameters could be re-estimated after each relevant CPT operation in 2009. This choice conforms to practical planning constraints, which demand that models are kept fixed, for instance, for periods of 12 months. The forecast study is restricted to the CPTs for which at least one factor is significant at the $1 \%$ significance level.

Three prediction methods are compared. The first is the method that is currently employed in the OR management of both hospitals. The predicted time is simply the average of the ten most recent durations of this CPT. The second method predicts the procedure time to be the median of the $3-\log \mathrm{N}$ distribution (without factors), that is, $\alpha+\exp (\beta)$. The third method predicts the case duration to be equal to the median of the ANOVA model, that is, $\alpha+\exp \left(\beta_{0}+\sum_{j} \beta_{j} F_{j}\right)$, including only those factors $F_{j}$ for which the estimate of $\beta_{j}$ is significant (at the $1 \%$ level). Predicted case durations are compared with the actual procedure times and the accuracy is evaluated in terms of absolute prediction errors (in minutes). The significance of the difference in mean absolute errors of two methods is tested using the paired $t$-test. 


\section{Results}

\subsection{Surgeon factors}

For each hospital and CPT, the significant surgeon factors are obtained using the backward selection strategy described in section 3.2. The results are summarized in Table 2, which shows how often each factor is found to be significant for significance levels of $10 \%, 5 \%$ and $1 \%$. For instance, in hospital A, the effect of the factor 'Gender' can be analyzed for 22 CPTs, as for the other seven CPTs the gender does not vary among the surgeons. The gender effect is significant (and negative) for three CPTs at the $10 \%$ level (with a median effect of $-1.8 \%$ ), for one CPT at the $5 \%$ level (with a median effect of $-8.2 \%$ ) and never at the $1 \%$ level. In hospital B, gender is never found to be significant, not even at the $10 \%$ level. This means that there is no support whatsoever for the commonly expressed opinion that female surgeons would work slower. The gender effect is very weak, and at most it indicates faster work of female surgeons.

Age effects are found to be often significant at the 5\% level, mostly with faster work of older surgeons, but the effect is significant at the $1 \%$ level only for two CPTs (with a time reduction of about $10 \%$ for older surgeons). Work rate effects are significant in several cases, with varying sign at levels of $10 \%$ and $5 \%$, but with a consistent time saving effect at the $1 \%$ level (of about $5 \%$ ) for high work rates. The team composition is significant in many cases, and in the far majority of cases the presence of a resident in the team causes longer procedure times (of about $15 \%$, at the $1 \%$ level). Daytime effects are significant in many cases, mostly with slower work in the afternoon.

Table 3 shows the estimated surgeon factor effects for each CPT separately, 29 for hospital A and 25 for hospital B. The effects are shown only if they are significant at the $10 \%$ level. The number of significant factors varies among CPTs. For each of the 22 CPTs that are performed at both hospitals, the sign and size of the effects are often quite the same in both hospitals, even though the effects of some factors

Table 2. Surgeon factor effects (number of CPTs with positive and negative effects, and median percentage effect on procedure time).

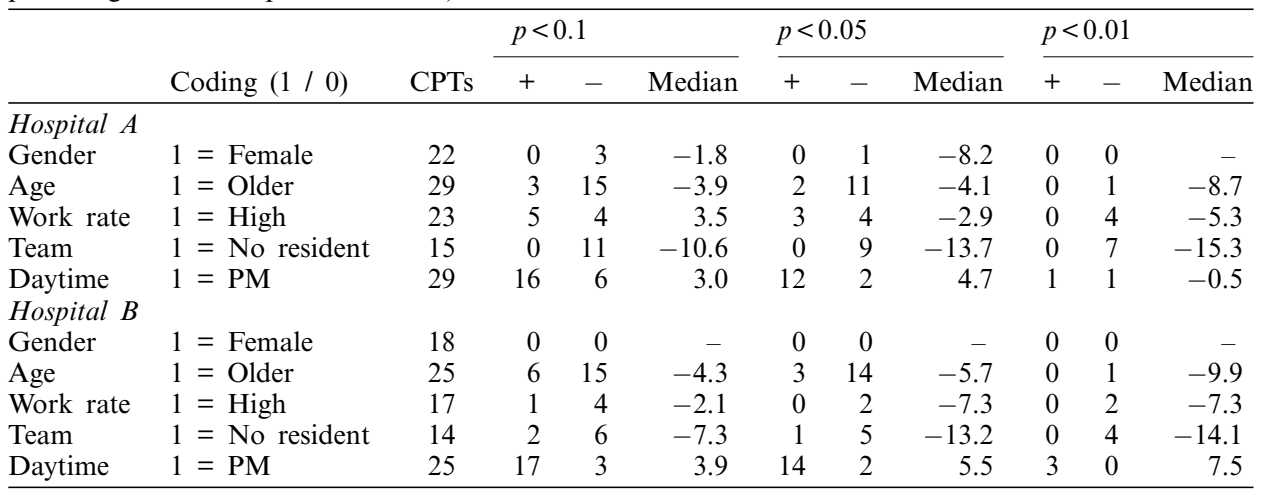

(c) 2009 The Authors. Journal compilation @ 2010 VVS. 


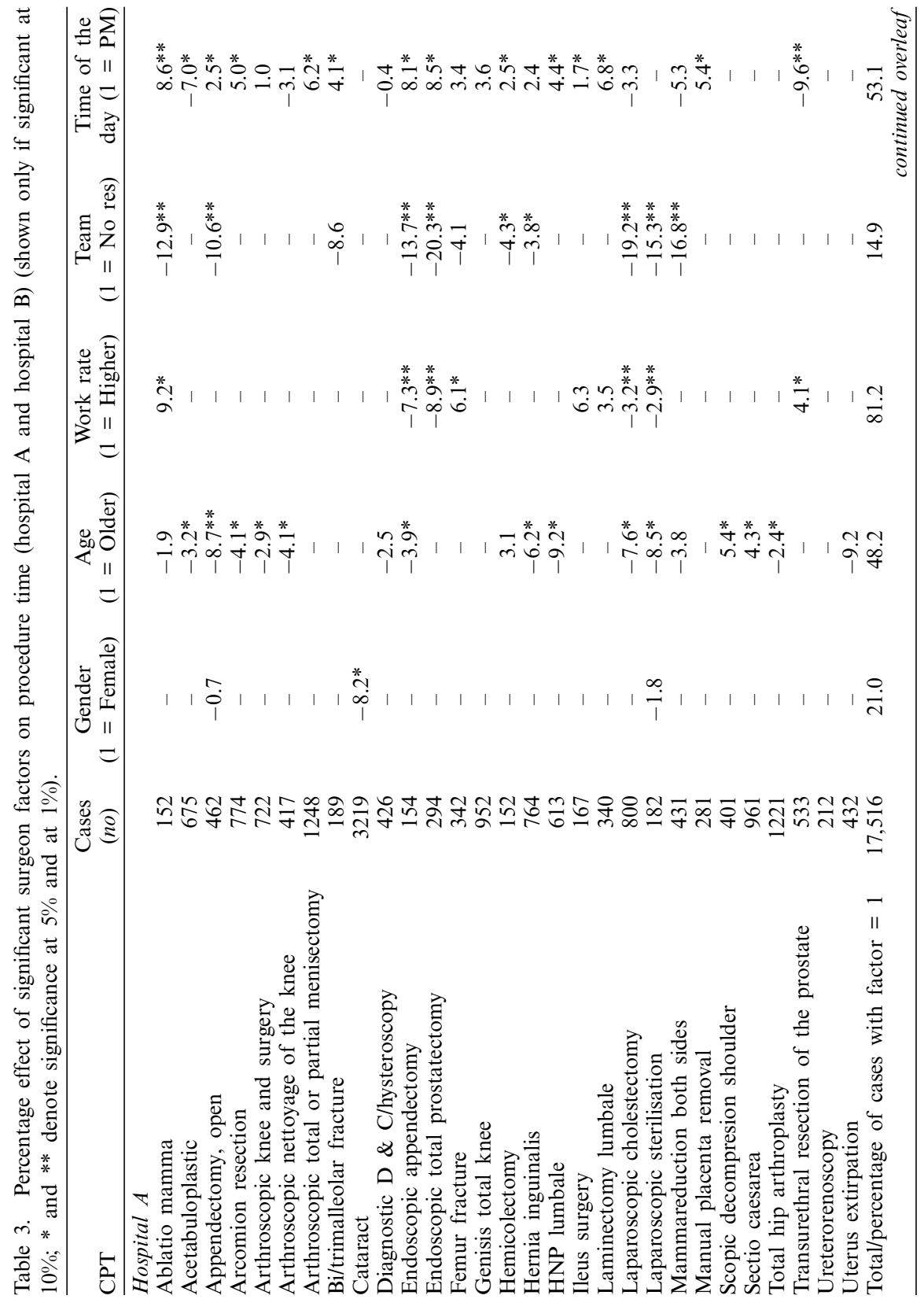




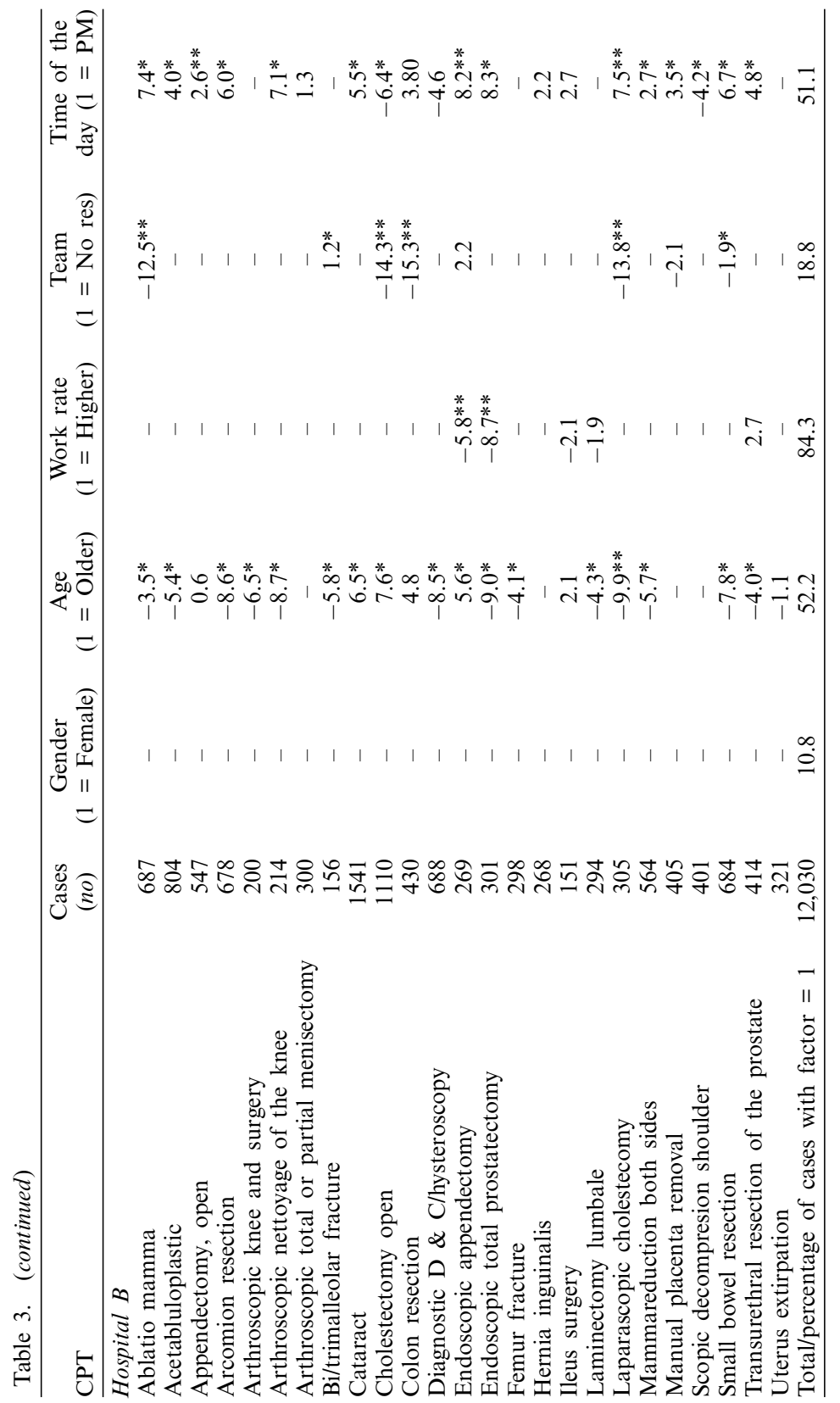


cannot be estimated at both hospitals, that is, if the factor does not vary for the CPT under consideration. For instance, for the CPT ablatio mamma, the age affect in hospitals $\mathrm{A}$ and $\mathrm{B}$ is respectively $-1.9 \%$ and $-3.5 \%$, the team effect is $-12.9 \%$ and $-12.5 \%$, the time of the day effect is $8.6 \%$ and $7.4 \%$ and the work rate effect is significant only for hospital A (at the 5\% level) and not for hospital B (at the $10 \%$ level).

Age and time of the day are the factors found most often to be significant. Work rate and team composition are also significant in many cases, and the largest percentage effects are found for these two factors. Gender is nearly never of any importance. The only significant gender effect at the $5 \%$ level is for cataract in hospital A, where female surgeons work $8 \%$ faster than male surgeons. The CPTs that have at least two significant factors at the $1 \%$ level correspond to relatively complicated surgical procedures requiring special skills: ablatio mamma, open appendectomy, endoscopic appendectomy, endoscopic total prostatectomy, laparoscopic cholestectomy and laparoscopic sterilization. For many of these complicated procedures, the work rate and team composition effects on procedure times are considerable, up to $20 \%$. Compared with less demanding CPTs, complex procedures require more time both for on-the-job training of residents and for activating specialized skills if the surgeon did not practice these skills within the preceding three weeks.

Summarizing, the largest effects are obtained for work rate and team composition for complicated CPTs. In most cases (and at the 1\% level always), procedure times are relatively shorter for older surgeons, for a high work rate and for teams without resident. Gender has hardly any effect. In most cases, procedure times are shorter in the morning than in the afternoon, but for some CPTs this effect is reversed.

The mixed time of the day effect can be due to the fact that this effect is measured jointly for the full OR team involved in the operation and without information on the time preference of the members of the team. A small-scale study was performed to investigate this further. Ten surgeons of hospital A and ten surgeons of hospital B were asked whether they have any preference for performing operations in the morning or in the afternoon. Of these 20 surgeons, nine prefer the morning, ten the afternoon, and one surgeon has no preference. In total, the 19 surgeons with a preference are active in 64 CPTs. For each surgeon and CPT, the average case duration in the morning is compared with that in the afternoon. Of the 64 surgeon-CPT combinations, the fastest work was delivered in 48 cases in the preferred time of the day and in 16 cases in the non-preferred time of the day. This effect of preferred time of the day on case durations is significant (the $P$-value according to the binomial distribution with a success probability of $50 \%$ is smaller than $0.01 \%$ ). For hospital A (B), the fastest work was delivered in 23 (25) cases in the preferred time of the day and in 7 (9) cases in the non-preferred time of the day, corresponding to a $P$-value for the absence of the time of the day effects of less than $1 \%$ in both cases.

As the time of the day preferences are not known for many of the surgeons involved in the CPTs of Table 1, this factor could not be incorporated in the analysis (C) 2009 The Authors. Journal compilation (c) 2010 VVS. 
of surgeon factor effects in Tables 2 and 3. However, the small-scale study indicates that it may help to incorporate surgeon preferences in OR planning.

\subsection{Prediction}

In order to evaluate the practical usefulness of surgeon factors in predicting case durations, the attention is restricted to CPTs for which at least one surgeon factor is significant at the $1 \%$ level. This holds true for eight CPTs in hospital A and seven CPTs in hospital B, five of which occur at both hospitals. The ANOVA models, estimated with the data of 2005-2008 and with the estimated factor effects of Table 3 that are significant at the $1 \%$ level, are used to predict the procedure times for the period from January to August 2009. The total number of predicted case durations is 683 for hospital A and 575 for hospital B.

Table 4 summarizes the results of three prediction methods, that is, the current method (average of last ten cases), the three-parameter lognormal model without factors $(3-\log N)$ and the ANOVA model. The table shows the mean and standard deviation of the absolute prediction errors, that is, the differences between the predicted time and the actual case duration. The differences in mean absolute prediction errors of the three methods are evaluated both in absolute terms (in minutes) and in relative terms (as percentage of the median procedure time for each CPT over the prediction period).

As an illustration, Figure 1 shows the absolute prediction errors and the differences of these errors of the three prediction methods for the 71 endoscopic appendectomy operations that took place in hospital A between January and August 2009. The current method predicts the procedure time as the average of the last ten case durations of this CPT and this estimate is updated after each operation in 2009. The $3-\log N$ predictions are obtained from the ANOVA model without factors, estimated with data from 2005 to 2008 and with fixed parameters for 2009. Finally, the ANOVA predictions are also obtained from a model estimated with data from 2005 to 2008 and with fixed parameters for 2009. This model includes factors only if they are significant at the $1 \%$ level. Table 3 shows that the included factors are work rate (with coefficient -0.073 ) and team composition (with coefficient -0.137 ). Figure 1 shows that the smallest prediction errors are obtained for ANOVA and that $3-\log N$ is the second best. The predictions of ANOVA are better than the current method in 67 of 71 cases and they are better than $3-\log N$ in 53 of 71 cases. The differences in absolute forecast errors of the three methods are all significant (at the $5 \%$ level) when tested using the paired $t$-test.

Table 4 shows that, in all of the considered 15 CPTs in hospitals A and B, the $3-\log \mathrm{N}$ predictions are more accurate than the currently employed method. The same holds true for the ANOVA predictions, except for transurethral resection of the prostate in hospital A. Compared with the current method, the forecast improvements of $3-\log \mathrm{N}$ are up to $10 \%$, and those of ANOVA are up to $18 \%$. The ANOVA predictions are better than the $3-\log N$ predictions in the far majority of cases (11 of 15 ), 


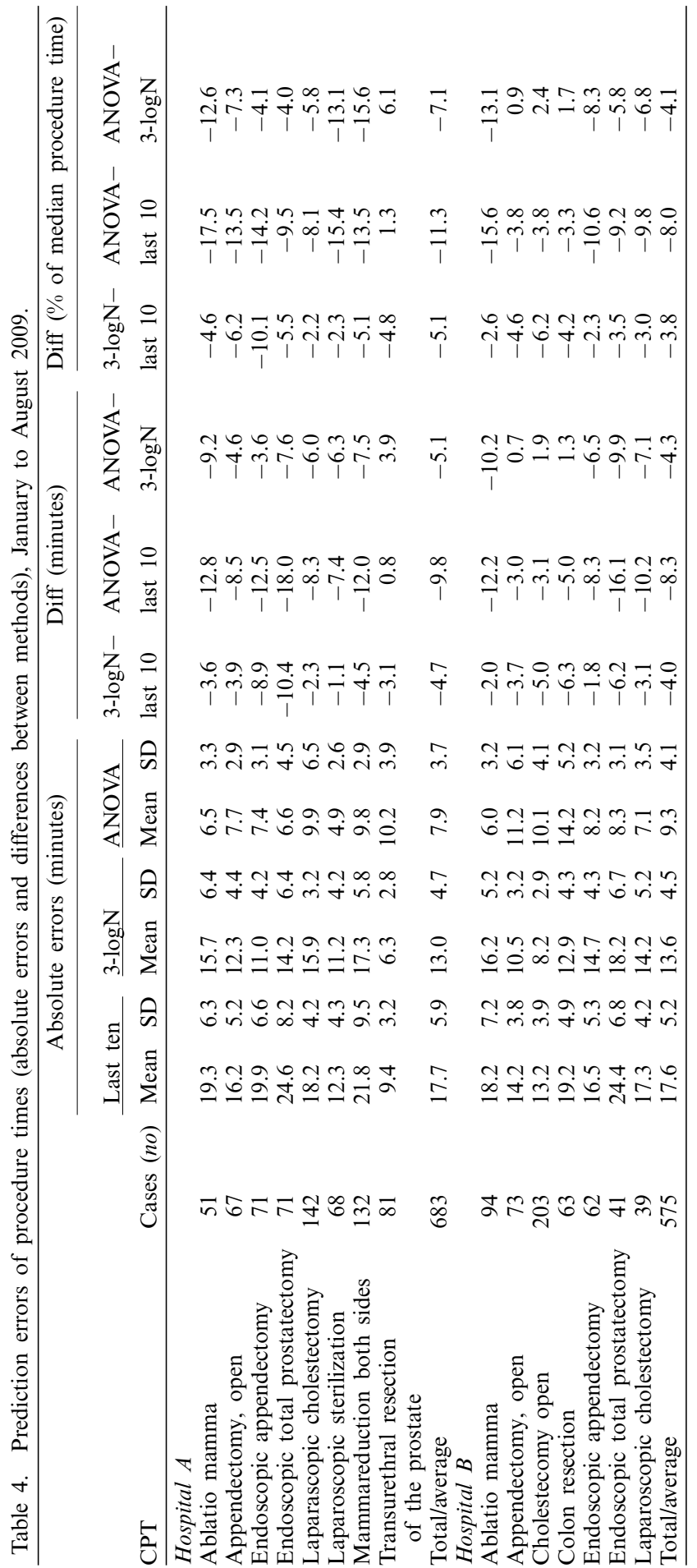



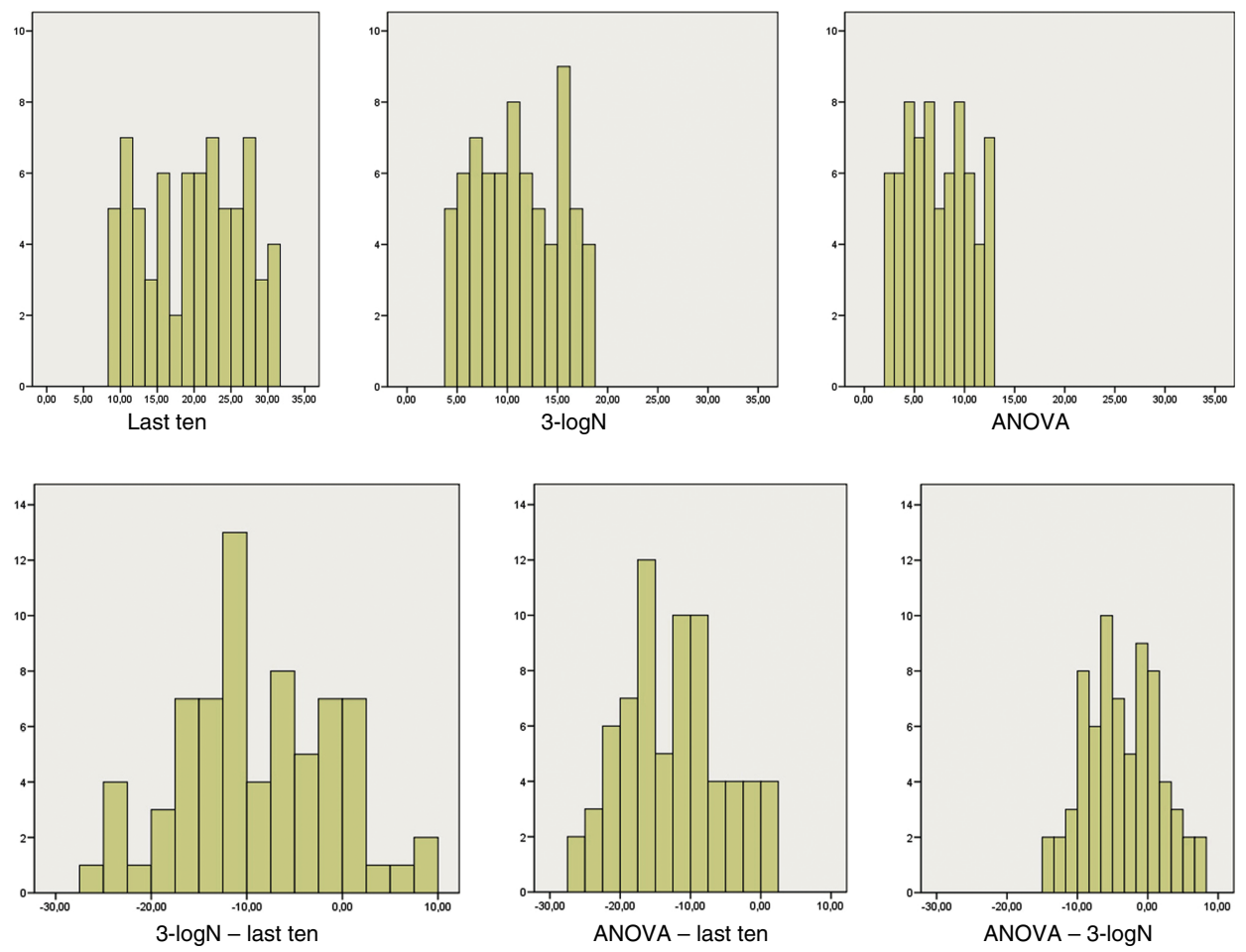

Fig. 1. Histograms of absolute forecast errors (top) and differences in absolute forecast errors (bottom) for 71 procedure times of endoscopic appendectomy.

with gains of up to $15 \%$. For three CPTs in hospital B, 3- $\log N$ is slightly better than ANOVA (up to $2 \%$ ), and for one CPT in hospital A, 3- $\log \mathrm{N}$ is $6 \%$ better than ANOVA. The paired $t$-test finds that, for hospital A, ANOVA improves significantly on $3-\log \mathrm{N}$ (at the $5 \%$ level) for seven of eight CPTs and the reverse holds true for the remaining CPT. For hospital B, ANOVA is significantly better than $3-\log N$ for four of seven CPTs and the difference is not significant for the other three CPTs.

When averaged over the eight considered CPTs in hospital A, the gain in prediction accuracy is 5 minutes $(5 \%)$ for $3-\log N$ compared with the current method, 10 minutes $(11 \%)$ for ANOVA compared with the current method and 5 minutes (7\%) for ANOVA compared with 3- $\log$. For hospital B, the prediction gains are 4 minutes $(4 \%)$ for $3-\log N$ compared with the current method, 8 minutes $(8 \%)$ for ANOVA compared with the current method and 4 minutes (4\%) for ANOVA compared with $3-\log \mathrm{N}$. On average, the standard deviation of the prediction errors is smallest for ANOVA (3.7 minutes in hospital A and 4.1 minutes in hospital B) compared with $3-\log \mathrm{N}$ (4.7 in A and 4.5 in B) and the current method (5.9 in A and 5.2 in B). Although these differences are not large, reduction in uncertainty is important in OR planning. It is a nice finding that the improved prediction accuracy of ANOVA, which is based on more elaborate models involving surgeon factors, is (C) 2009 The Authors. Journal compilation (C) 2010 VVS. 
combined with reduced forecast uncertainty. Stated otherwise, the smaller prediction bias of ANOVA comes without any cost of increased variance.

\section{Conclusion}

Depending on the type of operation (CPT) and on the hospital, procedure times may depend on several surgeon factors. In particular, for complex operations, factors like relevant work rate experience of the surgeon and composition of the surgical team may have large effects. The effect of team composition goes up to $20 \%$ and, when combined with work rate, the total effect goes up to $30 \%$. Other relevant factors are age of the surgeon and time of the day. Gender has nearly never any effect, and the only effect that is significant (at the $5 \%$ level) is found for cataract, where female surgeons work $8 \%$ faster than male surgeons. A predictive out-of-sample analysis for case durations in 2009 shows that surgeon factors help in predicting case durations. Compared with the methodology currently employed in both hospitals, mean absolute prediction errors are reduced by up to 18 minutes and up to $18 \%$ of the median procedure time.

The most significant gains are obtained for relatively complex CPTs, especially those involving endoscopic and laparoscopic procedures. As the complexity of surgical procedures shows an ever-increasing trend, surgeon factors may become even more important in the future.

The practical implementation of (ANOVA or other) prediction models is done best after consultation of surgeons, OR management and other staff involved in the operation room activities. As hospitals differ widely in aspects like surgical experience with different specializations, organizational structure, OR protocols and OR logistics, the effect of surgeon factors will differ among hospitals. Therefore, it may be best to estimate separate models for each hospital. The results of this paper show several differences between the two hospitals considered, although the type of effect is quite the same in many cases, especially for complex procedures.

The achieved improved forecast accuracy can be of great help for operation room planning. Reduction in case duration uncertainty will have positive benefits in terms of patient health care and human resource planning in hospitals.

\section{References}

BARNoOn, S. and H. Wolfe (1968), Scheduling a multiple operating room system: a simulation approach, Health Services Research 3, 272-285.

Dexter, F., E. U. Dexter, D. Masursky and N. A. Nussmeier (2008), Systematic review of general thoracic surgery articles to identify predictors of operating room case durations, Anesthesia Analgesia 106, 1232-1241.

Hancock, W. M., P. F. Walter, R. A. More and N. D. Glick (1988). Operating room scheduling data base analysis for scheduling, Journal of Medical Systems 12, 397-409. 
May, J. H., D. P. Strum and L. G. Vargas (2000), Fitting the lognormal distribution to surgical procedure times. Decision Sciences 31, 129-148.

Rossiter, C. E. and J. A. Reynolds (1963), Automatic monitoring of the time waited in outpatient departments, Medical Care 1, 218-225.

Spangler, W. E., D. P. Strum, L. G. Vargas and J. H. May (2004), Estimating procedure times for surgeries by determining location parameters for the lognormal model, Health Care Management Science 7, 97-104.

Stepaniak, P. S., C. Heis, G. H. H. Mannaerts, M. De Quelerij and G. De Vries (2009a), Modeling procedure and surgical times for CPT-anesthesia-surgeon combinations and evaluation in terms of case-duration prediction and operating room efficiency, Anesthesia Analgesia 109, 1232-1245.

Stepaniak, P. S., G. H. H. Mannaerts, M. De Quelerij and G. DE VRies (2009b), The effect of the operating room coordinator's risk appreciation on operating room efficiency, Anesthesia Analgesia 108, 1249-1256.

Strum, D. P., J. H. May and L. G. Vargas (2000a), Modeling the uncertainty of surgical procedure times: comparison of log-normal and normal models, Anesthesiology 92, 1160-1167.

Strum, D. P., A. R. Sampson, J. H. May and L. G. Vargas (2000b), Surgeon and type of anesthesia predict variability in surgical procedure times, Anesthesiology 92, 1454-1466.

Strum, D. P., J. H. May, A. R. Sampson, L. G. Vargas and W. E. Spangler (2003), Estimating times of surgeries with two component procedures: comparison of the lognormal and normal models, Anesthesiology 98, 232-240.

Tamm, A. S., O. Lagerquist, A. L. Ley and D. F. Collins (2009). Chronotype influences diurnal variations in the excitability of the human motor cortex and the ability to generate torque during a maximum voluntary contraction, Journal of Biological Rhythms 24, 211-224.

Van Houdenhoven, M. (2007), Healthcare logistics: The art of balance. Ph.D. Thesis, Erasmus University Rotterdam, Scriptum Publishers, Schiedam.

VISSERS, J. and R. BEECH (2005), Health operations management: patient flow logistics in health care, Routledge, Abingdon, Oxon.

Received: September 2009. Revised: September 2009. 